\title{
Interactions of hydrogen with amorphous hafnium oxide
}

\author{
Moloud Kaviani, ${ }^{,}, *$ Valeri V. Afanas’ev, ${ }^{2, \dagger}$ and Alexander L. Shluger ${ }^{1,3, \ddagger}$ \\ ${ }^{1}$ Advanced Institute for Materials Research (AIMR), Tohoku University, Sendai 980-8577, Japan \\ ${ }^{2}$ Department of Physics, University of Leuven, Celestijnenlaan 200D, B-3001 Leuven, Belgium \\ ${ }^{3}$ Department of Physics and Astronomy and London Centre for Nanotechnology, University College London, \\ Gower Street, London WC1E 6BT, United Kingdom
}

(Received 23 November 2016; published 8 February 2017)

\begin{abstract}
We used density functional theory (DFT) calculations to study the interaction of hydrogen with amorphous hafnia $\left(\mathrm{a}-\mathrm{HfO}_{2}\right)$ using a hybrid exchange-correlation functional. Injection of atomic hydrogen, its diffusion towards electrodes, and ionization can be seen as key processes underlying charge instability of high-permittivity amorphous hafnia layers in many applications. Hydrogen in many wide band gap crystalline oxides exhibits negative- $U$ behavior $(+1$ and -1 charged states are thermodynamically more stable than the neutral state) . Our results show that in a- $\mathrm{HfO}_{2}$ hydrogen is also negative- $\mathrm{U}$, with charged states being the most thermodynamically stable at all Fermi level positions. However, metastable atomic hydrogen can share an electron with intrinsic electron trapping precursor sites [Phys. Rev. B 94, 020103 (2016).] forming a $\left[e_{t r}^{-}+\mathrm{O}-\mathrm{H}\right]$ center, which is lower in energy on average by about $0.2 \mathrm{eV}$. These electron trapping sites can affect both the dynamics and thermodynamics of the interaction of hydrogen with a- $\mathrm{HfO}_{2}$ and the electrical behavior of amorphous hafnia films in CMOS devices.
\end{abstract}

DOI: 10.1103/PhysRevB.95.075117

\section{INTRODUCTION}

Thin films of amorphous (a) hafnium dioxide a- $\mathrm{HfO}_{2}$ are used for optical coating [1,2] and as the gate dielectric in complementary metal oxide semiconductor (CMOS) technology due to high dielectric constant and reliability [3,4]. Hafnia layers are also applied in resistive memory devices [5-7] and as the gate dielectric for thin film transistors (TFTs) based on metal oxide channel materials, such as indium zinc oxide and indium gallium zinc oxide [8-10]. However, charge instability of $\mathrm{Hf}$ oxide films is becoming an increasingly significant issue since it directly impacts electric field in the charge transport region [11]. In particular, bias-temperature instabilities related to both positive and negative charging of ultrathin hafnia layers in CMOS devices appear to increase exponentially as the film thickness decreases to the range of a few nanometers [11-14]. The charging-related reliability issues actually set the physical limit for the gate oxide scaling, however, despite huge practical significance, the origin of this charging behavior of a- $\mathrm{HfO}_{2}$ remains unknown. Initially, the oxide charging has been related to $\mathrm{O}$ vacancies [12]. However, positions of charge transition levels of oxygen vacancy in a- $\mathrm{HfO}_{2}[15,16]$ with respect to the experimental band offsets at interfaces of $\mathrm{HfO}_{2}$ with relevant semiconductors and metals [17] rule out this explanation urging one to consider alternative model(s) [16].

In this work we examine the interaction of hydrogen with a- $\mathrm{HfO}_{2}$ as a possible origin of charge instability in CMOS devices. Hydrogen is abundantly available during atomic-layer deposition of $\mathrm{HfO}_{2}$ and subsequent device processing. It can also be injected into the oxide during device operation and electrical stress from gate stack or from the spacer regions. Though technology primarily aims at passivation of existing

\footnotetext{
*moloud.kaviani@gmail.com

†valeri.afanasiev@fys.kuleuven.be

†a.shluger@ucl.ac.uk
}

defects by hydrogen to improve the device performance, it may also induce new defects or charges detrimental to the reliability. For example, most of the charging phenomena in much better studied insulating a-SiO${ }_{2}$ films are associated with hydrogen-related network fragments [18-21].

In a broader sense, hafnia is a representative of transition metal oxides with the structure and properties similar to a much better studied zirconia. The interaction of hydrogen with this class of materials is important for a wide range of applications and has been studied extensively, with hydrogen reduction of $\mathrm{Zr}$ and $\mathrm{Hf}$ oxides first reported more than 50 years ago [22]. Density functional theory (DFT) calculations played an important role in predicting the properties of hydrogen in crystalline oxides, including zirconia and hafnia [23-28]. In particular, previous investigations of thermodynamic stability and charge transition levels of hydrogen in stoichiometric monoclinic $\mathrm{HfO}_{2}$ and $\mathrm{ZrO}_{2}$ as a function of the Fermi level position using nonlocal DFT functionals concluded that $\mathrm{H}$ is a negative- $U$ system, with the +1 or -1 charge states lower in energy for all relevant Fermi-level values [23,28,29]. In the positive charge state, the hydrogen interstitial is bonded to a threefold-coordinated oxygen atom, with a bond length of $0.98 \AA$. Negatively charged $\mathrm{H}^{-}$ion sits interstitially and is coordinated by two [28] or three Hf atoms [29]. Such behavior is characteristic to many crystalline wide-gap oxides [28]. However, the properties of $\mathrm{H}$ in amorphous $\mathrm{HfO}_{2}$ have not yet been studied theoretically and are the focus of this work.

The dynamics of interaction of hydrogen with crystalline oxides is complex and its understanding has been aided tremendously by the so-called oxide muonics, where muonium is used as a pseudoisotope of hydrogen, as nicely summarized in Refs. [30,31]. In a nutshell, hydrogen atom injected into in wide gap perfect bulk oxide can be (meta)stable and diffuse through the lattice. If the so-called $+/-$ pinning level $[28,32]$ is above the bottom of the conduction band or the band gap is smaller than about $5.5 \mathrm{eV}$, atomic $\mathrm{H}$ will eventually donate an electron into the conduction band and become a proton 
and bind to the lattice oxygen ion. If there are defects in bulk material, such as oxygen vacancies, grain boundaries, misfit dislocations $[33,34]$, atomic hydrogen can donate its electron to these defects and again form an $\mathrm{O}-\mathrm{H}$ bond at one of the lattice sites. If the Fermi level set by external electrodes or dopants is high enough, it will eventually trap an extra electron and become $\mathrm{H}^{-}$.

At a closer look, the picture is more nuanced as it depends on the character and concentration of electron trapping sites in a particular system. For example, atomic hydrogen does not react with terraces at the $\mathrm{MgO}$ (001) surface, but donates electrons to three-coordinated $\mathrm{Mg}$ sites at corners and kinks at that surface, creating new $\left[\mathrm{Mg}^{+}-\mathrm{O}-\mathrm{H}\right]$-type centers $[35,36]$. The recent study of amorphous silica has concluded that, unlike in $\alpha$ quartz, atomic $\mathrm{H}$ can break some strained $\mathrm{Si}-\mathrm{O}$ bonds and form a new thermodynamically stable defect, termed hydroxyl $\mathrm{E}^{\prime}$ center $[37,38]$. In this case, the electron is localized on a $\mathrm{Si}$ atom and a proton forms an $\mathrm{O}-\mathrm{H}$ bond nearby. However, the proton is loosely bound and the volatility observed in electrical measurements of $\mathrm{SiO}_{2}$-based CMOS devices can be explained by the motion of the proton from one $\mathrm{O}$ ion to another [39]. Thus structural disorder adds important new features to the interaction of hydrogen with materials.

Amorphous $\mathrm{HfO}_{2}$ has a wider distribution of $\mathrm{O}$ and Hf coordinations than monoclinic (m)- $\mathrm{HfO}_{2}$. It has been recently demonstrated that low-coordinated $\mathrm{Hf}$ atoms and those associated with long $\mathrm{Hf}-\mathrm{O}$ bonds in a- $\mathrm{HfO}_{2}$ can trap extra electrons forming deep single- and bi-electron polaron states [40]. These electron trapping sites can affect both the dynamics and thermodynamics of the interaction of hydrogen with $\mathrm{HfO}_{2}$, as confirmed by the results presented below. Our results demonstrate that charged hydrogen states remain the most thermodynamically stable in a- $\mathrm{HfO}_{2}$ at all Fermi level positions. However, metastable atomic $\mathrm{H}$ can have two configurations, $\mathrm{H}_{i}^{0}$ and $\left[e_{t r}^{-}+\mathrm{O}-\mathrm{H}\right]$, with the latter configuration being slightly more stable. We discuss how these states affect the electrical behavior of amorphous hafnia films in CMOS devices.

\section{COMPUTATIONAL METHODS}

The fact that atomic hydrogen in oxides can adopt two different configurations: $\mathrm{H}_{i}^{0}$ and $\left[e^{-}+\mathrm{O}-\mathrm{H}\right]$ in the same material, has been observed and discussed in experimental studies and theoretical calculations, e.g., [34,41]. These configurations are connected via a potential barrier and which one is more energetically favorable depends on the band gap [41-43]. In the context of this work, the dissociation of atomic $\mathrm{H}$ into an electron polaron and a nascent $\mathrm{O}-\mathrm{H}$ group in a range of Fermi level positions has been suggested in stabilized cubic $\mathrm{ZrO}_{2}[25,44]$. On the other hand, calculations using GGA functionals, which underestimate the band gap, have concluded that the $\mathrm{H}$ atom is an electron donor in the full Fermi level range, e.g., [24,27,42], i.e., donates its electron to the conduction band and becomes a proton forming an $\mathrm{O}-\mathrm{H}$ bond. The $\mathrm{H}_{i}^{0}$ and $\left[e^{-}+\mathrm{O}-\mathrm{H}\right]$ configurations of atomic $\mathrm{H}$ in monoclinic $\mathrm{HfO}_{2}$ and $\mathrm{ZrO}_{2}$ have been observed separately in Refs. [28,42], as a result of the difference in the band gap. Whether the $\mathrm{H}_{i}^{0}$ or $\left[e^{-}+\mathrm{O}-\mathrm{H}\right]$ state is lower in energy in a particular system thus strongly depends on the band gap value and whether polaron states or other electron traps exist in the system. Theoretical predictions therefore depend strongly on whether the density functional used can correctly reproduce the band gap value and polaron trapping, as demonstrated by the controversy of results in Refs. [24,25,42].

In this work we used the methodology described in our recent paper [40]. To create initial models a- $\mathrm{HfO}_{2}$ structures we used pair potentials developed in Ref. [45] and the LAMMPS package [46]. Nine periodic models of a- $\mathrm{HfO}_{2}$, each containing 324 atoms, have been generated using classical molecular dynamics and a melt and quench procedure in an NPT ensemble, as discussed in more detail in Ref. [40]. The densities of the produced models are ranging from 8.6 to $9.2 \mathrm{~g} \mathrm{~cm}^{-3}$. These models exhibit wide distributions of bond lengths and atomic coordinations [40].

Further optimization of the volume and geometry of these structures was performed using DFT as implemented in the CP2K code $[47,48]$ with the PBE0-TC-LRC functional [47] and an exchange cutoff radius of $4.0 \AA$. The CP2K code employs a Gaussian basis set mixed with an auxiliary plane-wave basis set [49]. Polarized double- $\zeta$ valance basis sets [50] were employed for all atoms in conjunction with the GTH pseudopotential [51]. To reduce the computational cost of nonlocal functional calculations, the auxiliary density matrix method (ADMM) was employed [47]. All geometry optimizations were performed using the Broyden-Fletcher-Goldfarb-Shanno (BFGS) optimizer to minimize forces on atoms to $2.3 \times$ $10^{-2} \mathrm{eV} \AA^{-1}$ threshold.

The densities of the nine DFT optimized structures used in this work are in the range of $9.6-9.7 \mathrm{~g} \mathrm{~cm}^{-3}$ which is justified by inhomogeneity of doped and partially crystallized thin a- $\mathrm{HfO}_{2}$ films (see, e.g., [52-54]). The topology of a- $\mathrm{HfO}_{2}$ models obtained using classical MD simulations does not change as a result of DFT geometry optimization of the volume and atomic structures. The distributions of $\mathrm{Hf}-\mathrm{O}$ and Hf-Hf bond lengths obtained after the DFT cell and geometry optimization of neutral cells are discussed in Ref. [40]. Hf ions are 5-7 coordinated and $\mathrm{O}$ ions are 2-4 coordinated. We note that in the $\mathrm{m}-\mathrm{HfO}_{2}$ the $\mathrm{Hf}$ atoms are sevenfold coordinated and the oxygen atoms are three- and fourfold coordinated. The average Hf-O bond length is $2.1 \AA$ (ranging from 1.95 to $2.35 \AA$ ), which is very close to the $\mathrm{Hf}-\mathrm{O}$ bond lengths in $\mathrm{m}-\mathrm{HfO}_{2}$ (around $2.1 \AA$ ). The one-electron band gap of a- $\mathrm{HfO}_{2}$ structures does not contain localized states due to under-coordinated atoms and is equal to $6.0 \mathrm{eV}$, on average. However, both the top of the valence band and the bottom of the conduction band are determined by partially localized oxygen $p$ and hafnium $d$ electronic states, respectively, characteristic to disordered systems and similar to those observed in a-SiO [55].

As demonstrated in Ref. [40], we observe spontaneous localization of one and two extra electrons in deep states in each considered system. In $60 \%$ cases precursor sites for this localization are the $\mathrm{Hf}$ atoms which have at least three oxygen neighbors with the distance longer than $2.16 \AA$. In around one-third of the cases, the extra electron is localized by five-coordinated $\mathrm{Hf}$ atoms, which also have longer $\mathrm{Hf}-\mathrm{O}$ bonds. The average position of the Kohn-Sham (KS) level for the electron polaron in these structures is $2.10 \mathrm{eV}$ ranging from 


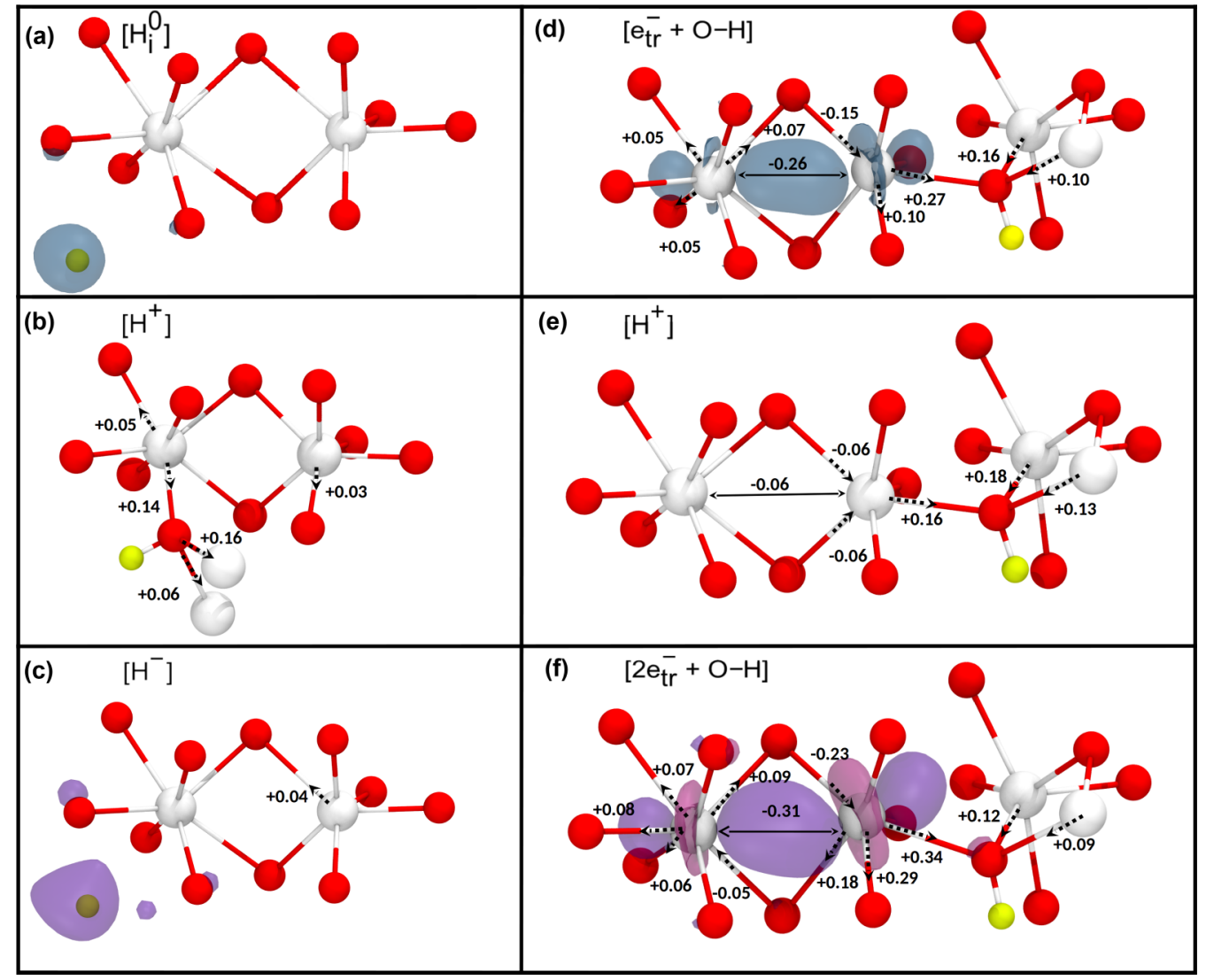

FIG. 1. Geometric configurations of hydrogen interstitial and $\left[e_{t r}^{-}+\mathrm{O}-\mathrm{H}\right]$ centers in different charge states in a-HfO $\mathrm{H}_{2}$. (a) Neutral interstitial $\mathrm{H}_{i}^{0}$; (b) positively charged $\mathrm{H}^{+}$; (c) negatively charged $\mathrm{H}^{-}$; (d) configuration of neutral $\left[e_{t r}^{-}+\mathrm{O}-\mathrm{H}\right]$ center; (e) configuration of positively charged $\left[e_{t r}^{-}+\mathrm{O}-\mathrm{H}\right]$ center; (f) configuration of negatively charged $\left[2 e_{t r}^{-}+\mathrm{O}-\mathrm{H}\right]$ center. The ionic displacements (with respect to the original positions of ions in a- $\mathrm{HfO}_{2}$ ) that are less than $0.02 \AA$ are not shown in (a)-(c) and those less than $0.05 \AA$ in (d) and (e). The dashed arrows show the directions of larger ion displacements. The square modulus of HOMO is shown in (a) with the isovalue of 0.028 and in (c) with the isovalue of 0.1 , in (d) with the isovalue of 0.01 , and in (e) with the isovalue of 0.07 .

1.6 to $2.4 \mathrm{eV}$ below the bottom of the conduction band, whereas for bi-polarons it is $1.93 \mathrm{eV}$, ranging from 1.10 to $2.60 \mathrm{eV}$ below the bottom of the conduction band. Roughly about $8 \%$ of all $\mathrm{Hf}$ sites serve as precursors for electron trapping. As we show below, these states can compete for electrons with hydrogen. We use several structures in further calculations in order to sample different defect environments and to be able to build an adequate statistics of hydrogen defects affected by polaron trapping in a- $\mathrm{HfO}_{2}$. Each 324 atom cell contains up to four intrinsic precursor sites for the electron trapping and nine cells are used to sample more such sites. The differences in formation energies and defect level positions between different $\mathrm{H}$ positions within the same model are comparable to those between different models.

The formation energies of hydrogen defects (D) in the charge state $q$ were calculated as

$$
\begin{aligned}
E_{f}^{D, q}\left(\epsilon_{F}\right)= & E_{\text {defect }}^{D, q}-\left(E_{\text {bulk }}+E_{H}^{0}\right) \\
& +q\left(E_{V}+\epsilon_{F}+\delta V\right)+E_{\text {corr }},
\end{aligned}
$$

where $E_{\text {defect }}^{D, q}$ is the total energy of the defect system with charge $q, E_{\text {bulk }}$ is the total energy of the unperturbed host, and $E_{H}^{0}$ is the energy of a $\mathrm{H}$ atom. $E_{V}$ is referred to the valance band maximum (VBM) and $\delta V$ is a potential alignment term. $E_{\text {corr }}$ is a correction term for the periodic interaction between the localized charges in charged systems. The Lany and Zunger correction method for charge correction was used [56,57] with the dielectric constant of 22 [58] for amorphous hafnia.

Recent calculations of protonic defects in a range of perovskites [59-61] highlighted the role of configurational and vibrational entropy in formation energies of these defects, especially at high temperatures. We neglect this effect here as it has been shown to be relatively small for O-H defects [59] and at temperatures relevant for device anneal and operation.

\section{RESULTS OF CALCULATIONS}

Our starting assumption is that atomic $\mathrm{H}$ can diffuse through a- $\mathrm{HfO}_{2}$ exploring different sites. Therefore we placed a single $\mathrm{H}$ atom in different random positions, both close $(\sim 1.1 \AA)$ to the oxygen atoms connected to the electron trapping precursor sites and far from them, in nine a- $\mathrm{HfO}_{2}$ samples creating 60 initial configurations [52-54].

The geometry optimization demonstrates that, dependent on the local environment, the $\mathrm{H}$ atom can form two stable configurations: an interstitial $\mathrm{H}\left(\mathrm{H}_{i}^{0}\right)$ similar to the one in $\mathrm{m}-\mathrm{HfO}_{2}[23,28]$ and another configuration, which we termed $\left[e_{t r}^{-}+\mathrm{O}-\mathrm{H}\right]$, where $\mathrm{H}$ donates its electron to an electron trapping precursor site and the ensuing proton forms an $\mathrm{O}-\mathrm{H}$ 
bond with one of the $\mathrm{O}$ ions. This is essentially a chemical reaction and we compare the energies of the initial $\mathrm{H}_{i}^{0}$ and the final $\left[e_{t r}^{-}+\mathrm{O}-\mathrm{H}\right]$ states for each such reaction.

$\mathrm{H}_{i}^{0}$ weakly interacts with a- $\mathrm{HfO}_{2}$ matrix, with nearest neighbor $\mathrm{O}$ ions found at about $2.0 \AA$. The spin density of the system is almost completely localized on the $\mathrm{H}$ atom [see Fig. 1(a)] with an average Mulliken spin moment of 0.81 , ranging from 0.71 to 0.85 . The $\mathrm{H}$ atom introduces a deep one-electron level in the a- $\mathrm{HfO}_{2}$ gap ranging from 1.2 to $0.2 \mathrm{eV}$ above the valence band averaging at $0.7 \mathrm{eV}$. This level position is similar to the one obtained for cubic $\mathrm{ZrO}_{2}$ using the HSE functional in Ref. [25]. $\mathrm{H}_{i}^{0}$ can sit in any of the voids in a- $\mathrm{HfO}_{2}$, however, the total energy of the system depends on the local environment and is typically spread over $0.4 \mathrm{eV}$ for particular sample density.

In the positive charge state, the interstitial $\mathrm{H}$ undergoes large relaxation and the finial configuration is similar to that predicted in $\mathrm{m}-\mathrm{HfO}_{2}[23,28]$, as illustrated in Fig. 1(b). In the most stable configuration the proton interstitial binds to a threefold-coordinated oxygen atom, with a bond length of $\sim 0.98 \AA$. The energy of this defect depends even stronger on the local environment, as it involves a significant relaxation of surrounding ions, and is spread over about $1.0 \mathrm{eV}$ for particular sample density.

In the negative charge state $\mathrm{H}^{-}$, the extra electron is trapped by the $\mathrm{H}$ atom. $\mathrm{H}^{-}$interacts strongly with two or three $\mathrm{Hf}$ atoms, as shown in Fig. 1(c). The distance between the Hf and $\mathrm{H}$ is averaging at $2.0 \AA$, ranging from 1.5 to $2.2 \AA$. This defect introduces a one-electron level at $0.50 \mathrm{eV}$ above the a- $\mathrm{HfO}_{2}$ valence band, ranging from 0.05 to $1.03 \mathrm{eV}$ dependent on the sample density and local environment.

The $\left[e_{t r}^{-}+\mathrm{O}-\mathrm{H}\right]$ center represents the electron trapped at a precursor site and a nascent $\mathrm{O}-\mathrm{H}$ bond. It is typically formed by about $1.1 \AA$ displacement of $\mathrm{H}$ over a barrier from an interstitial position into an $\mathrm{O}-\mathrm{H}$ bond position. The value of this barrier is about $0.7 \mathrm{eV}$ and strongly depends on the local environment. However, this reaction is accompanied by the electron transfer and is nonadiabatic and not amenable to NEB calculations. More accurate prediction of this barrier is out of the scope of this work.

The electron is predominantly localized on two Hf ions with the square modulus of the wave function shown in in Fig. 1(d). The O-H bond averages at $0.99 \AA$ and shows a distribution from 0.97 to $1.08 \AA$. Due to the electron localization and the $\mathrm{O}-\mathrm{H}$ bond formation, the $\left[e_{t r}^{-}+\mathrm{O}-\mathrm{H}\right]$ center significantly deforms the surrounding network. The two Hf ions trapping the electron displace closer to each other by about $0.3 \AA$ whereas $\mathrm{Hf}-\mathrm{O}$ bonds connected to the hydrogen atom are significantly elongated [see Fig. 1(d)]. If the initial $\mathrm{Hf}-\mathrm{O}$ bonds are shorter than $2.16 \AA$, after the relaxation they elongate by about $0.15 \AA$ while the strained $\mathrm{Hf}-\mathrm{O}$ bonds (those which are longer than the $2.16 \AA$ ) become effectively broken. In some cases this creates structures which consist of a fivefold coordinated $\mathrm{Hf}$ atom facing a hydroxyl group. The remaining Hf-O bonds of the Hf atom connected to the hydroxyl bond and the Hf atom next to it elongate on average by about $0.12 \AA$, as shown in Fig. 1(d). The distance between the Hf and oxygen atoms, from which it dissociated, averages at $2.44 \AA$, showing a very wide distribution from 2.35 to $2.49 \AA$.
The electron donated by the $\mathrm{H}$ atom is highly localized on the precursor Hf atoms. The average of the spin moment is 0.90 ranging from 0.80 to 0.98 . These results are in line with our previous studies on the electron trapping in a- $\mathrm{HfO}_{2}$ [40]. This defect introduces a one-electron level at $2.3 \mathrm{eV}$ below the bottom of the a- $\mathrm{HfO}_{2}$ conduction band, ranging from 1.8 to $3.0 \mathrm{eV}$, dependent on the sample density and local environment. Within particular density samples the position of this level ranges within $0.5 \mathrm{eV}$. This state is mainly hafnium " $d$ " in character with a contribution from oxygen " $p$ " orbitals. The average position of this level is very similar to the one found for cubic $\mathrm{ZrO}_{2}$ in Ref. [25].

We note that the interaction between the trapped electron polaron and the proton forming the $\mathrm{O}-\mathrm{H}$ group is weak and we observe the creation of the $\left[e_{t r}^{-}+\mathrm{O}-\mathrm{H}\right]$ pairs at longer distances where an electron is transferred from the $\mathrm{H}$ atom to the precursor site by tunneling. These configurations are about $0.2 \mathrm{eV}$ higher in energy confirming the weak interaction between the polaron and $\mathrm{O}-\mathrm{H}$ screened by the amorphous hafnia. Taking the large concentration of electron trapping precursor sites, the $\left[e_{t r}^{-}+\mathrm{O}-\mathrm{H}\right]$ forming reaction is quite ubiquitous. However, this defect can exhibit volatility discussed for a similar defect in $\mathrm{a}_{-} \mathrm{SiO}_{2}$ in Ref. [39].

In the positive charge state, an electron leaves the precursor state, accompanied by strong local relaxation, and in the most stable configuration the proton remains bonded to an oxygen atom in the Hf-O-Hf bridge, as illustrated in Fig. 1(e). This configuration is identical to $\mathrm{H}^{+}$in Fig. 1(b). The $\mathrm{H}-\mathrm{O}$ bond length remains the same as in the neutral charge state ( $\sim 0.99 \AA)$. There is no barrier for transforming into this configuration after the electron emission from the $\left[e_{t r}^{-}+\mathrm{O}-\mathrm{H}\right]$ center.

In the negatively charged state, an extra electron is trapped by the precursor site forming an electron bi-polaron [40] and what could be termed a $\left[2 e_{t r}^{-}+\mathrm{O}-\mathrm{H}\right]$ center. In this case, the defect undergoes an even larger atomic relaxation with respect to the neutral precursor state. The distance between the two Hf ions trapping the two electrons decreases further whereas the distance between the oxygens and the Hf atoms, on which the two extra electrons are localized, increases. The electron trapping is the reason of the elongated $\mathrm{Hf}-\mathrm{O}$ bonds after the relaxation, as described in Ref. [40]. The one-electron level introduced in the gap by this defect is on average at $2.15 \mathrm{eV}$ below the bottom of the a- $\mathrm{HfO}_{2}$ conduction band, ranging from 1.4 to $2.8 \mathrm{eV}$.

To investigate the thermodynamic stability of these defects, we have calculated the formation energies of nine different hydroxyl and hydrogen interstitial configurations as a function of the Fermi level position of the system according to Eq. (2) in different charge states. Four representative formation energy plots are shown in Fig. 2. They demonstrate that, similar to $\mathrm{m}-\mathrm{HfO}_{2}$, neutral $\mathrm{H}_{i}$ is a negative-U state. The $\mathrm{H}^{+}$state dominates in the energy range up to $4.0 \mathrm{eV}$ above the top of the valence band. In most cases the $\left[e_{t r}^{-}+\mathrm{O}-\mathrm{H}\right]$ configuration in $\mathrm{a}-\mathrm{HfO}_{2}$ is slightly more thermodynamically stable than $\mathrm{H}_{i}^{0}$. The $\left[2 e_{t r}^{-}+\mathrm{O}-\mathrm{H}\right]$ center energy is always higher than that of $\mathrm{H}^{-}$. There is an energy barrier for transformation between these two configurations, but this nonadiabatic process accompanied by transfer of two electrons is not considered here. 

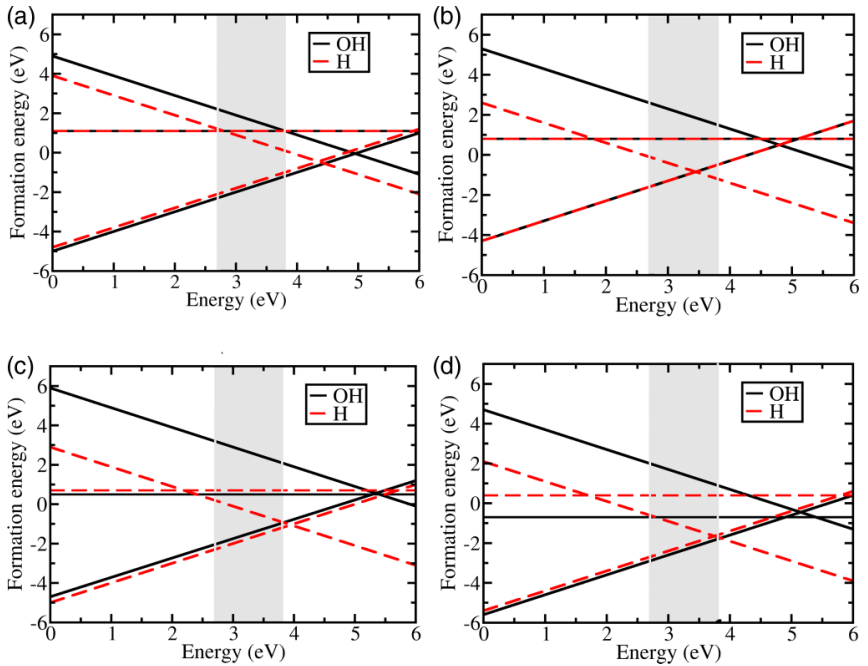

FIG. 2. Defect formation energy versus the Fermi level (with respect to the top of the a- $\mathrm{HfO}_{2}$ valence band) for four different configurations of the hydroxyl and hydrogen interstitial centers. The position of the $\mathrm{Si}$ band gap is in the $\mathrm{Si} / \mathrm{HfO}_{2}$ stack is shown by the shaded region. Formation energies corresponding to the $\left[e_{t r}^{-}+\mathrm{O}-\mathrm{H}\right]$ center are drawn as solid black lines while the formation energies of interstitial $\mathrm{H}$ configurations are drawn as dashed red lines.

\section{DISCUSSION AND CONCLUSIONS}

Let us first put these results in perspective with other similar bulk oxides, where hydrogen can be introduced during growth, chemical processes, radiation-induced decomposition of water or another type of hydrogen treatment. In this scenario, the Fermi level is either at the top of the valence band or is controlled by doping. The thermodynamically stable state of hydrogen in this case is $\mathrm{H}^{+}$and the hydrogen atom is metastable until it finds an acceptor to donate an electron and becomes $\left[e_{t r}^{-}+\mathrm{O}-\mathrm{H}\right]$. This is consistent with the muonium data for $\mathrm{m}-\mathrm{ZrO}_{2}$ and $\mathrm{m}-\mathrm{HfO}_{2}$ [62] demonstrating that atomic hydrogen should exist up to $700 \mathrm{~K}$. The temperature behavior of the muonium signal is consistent with the single electron level position of $\mathrm{H}_{i}^{0}$ in $\mathrm{m}-\mathrm{HfO}_{2}$ at about $0.7 \mathrm{eV}$ above the top of the valence band. This leads to the second electron trapping from the valence band at $700 \mathrm{~K}$ with the thermal activation energy of $0.4 \mathrm{eV}$, as observed in [62].

In a-HfO $\mathrm{H}_{2}$, however, atomic hydrogen can share an electron with an electron trapping precursor. Our calculations suggest that the $\mathrm{H}_{i}^{0}$ and $\left[e_{t r}^{-}+\mathrm{O}-\mathrm{H}\right]$ states are almost degenerate with the latter configuration more favorable by on average about 0.2 $\mathrm{eV}$. We note that this result is very sensitive to the band gap value, which in our calculations is close to the experimental value of $5.9 \mathrm{eV}$ [63]. This behavior can be understood using a relation:

$$
\Delta E=E_{\text {ion }}-E_{\text {polar }}-E_{\text {rel }},
$$

where $\Delta E$ is the energy difference between the interstitial $\mathrm{H}_{i}^{0}$ and $\left[e^{-}+\mathrm{O}-\mathrm{H}\right]$ states in the system. If the interstitial atomic $\mathrm{H}$ produces a level in the band gap, $\mathrm{E}_{\text {ion }}$ is the vertical ionization energy of that level into the conduction band to form $e^{-}+\mathrm{H}^{+}$; $\mathrm{E}_{\text {polar }}$ is the energy gain in trapping of an electron in a polaron or another trap state (grain boundary, impurity, etc.); $E_{\text {rel }}$ is the relaxation energy difference between the $\mathrm{H}$ and $\mathrm{O}-\mathrm{H}$ states. This relation clearly shows that whether the $\mathrm{H}_{i}^{0}$ or $\left[e^{-}+\mathrm{O}-\mathrm{H}\right]$ state is lower in a particular system strongly depends on the band gap value and whether polaron states or other electron traps exist in the system. In the case of a- $\mathrm{HfO}_{2}$, the ionization energy of the $\mathrm{H}$ atom into the conduction band is about $5.5 \mathrm{eV}$, the average electron trapping energy into a polaron state is about $1.0 \mathrm{eV}$ [40], both calculated as the total energy differences between the corresponding states. A proton undergoes a displacement from the $\mathrm{H}_{i}^{0}$ into the $\left[e^{-}+\mathrm{O}-\mathrm{H}\right]$ state of about $1.1 \AA$. The corresponding energy gain purely due to the $\mathrm{O}-\mathrm{H}$ formation can be estimated from molecular $\mathrm{OH}^{-}$ potential energy curves [64] for this displacement as about $4.1 \mathrm{eV}$. The rest of the balance is determined by the additional network relaxation caused by this reaction and is therefore very sensitive to the local environment. In monoclinic $\mathrm{HfO}_{2}$, the polaron trapping energy is about $0.3 \mathrm{eV}$ [65] and atomic hydrogen is stable until $700 \mathrm{~K}$.

In the context of microelectronic devices, the picture is richer. Here the Fermi level is controlled by the presence of external electrodes and applied bias. Hydrogen can enter thin oxide films from the surrounding metal electrodes during anneal [66]. If hydrogen enters the film as an atom, it can diffuse inside, exchange electron or hole with an electrode and diffuse as a proton or $\mathrm{H}^{-}$. It can also react at an electron precursor site and turn into $\left[e_{t r}^{-}+\mathrm{O}-\mathrm{H}\right]$ and then exchange electron or hole with an electrode, dependent on the band offset with the electrodes and bias applied. The $\left[e_{t r}^{-}+\mathrm{O}-\mathrm{H}\right]$ center is loosely bound and the proton can diffuse away, which effectively corresponds to the fact that at this Fermi level position $\mathrm{H}^{+}$is the most energetically favorable state. These types of processes contribute to bias temperature instability as well as random telegraph noise and other reliability issues in CMOS devices, as discussed in Refs. [39,67]. All in one, injection of atomic hydrogen into the oxide, its diffusion towards electrodes and subsequent ionization can be seen as key processes underlying charge instability of high-permittivity metal oxide layers. This picture finds substantial support from several experimental observations correlating oxide charging with the amount of atomic hydrogen released during charge injection [68,69]. Importantly, these processes depend on the mobility of hydrogen in different charge states, electron tunneling rates, and hence the oxide thickness.

There is not much information about mobility of hydrogen species in $\mathrm{HfO}_{2}$ [66], but much more is known for crystalline $\mathrm{ZrO}_{2}[25,70]$, which has very similar lattice parameters and other properties. There it is well established that the proton can move relatively fast via the so-called Grotthuss-type diffusion process of hopping between oxygens with barriers around $0.3 \mathrm{eV}$ [24]. A similar barrier has recently been calculated in $\mathrm{m}-\mathrm{HfO}_{2}$ [71]. The experimental data for atomic hydrogen is much more scattered and range between 0.2 and $2.0 \mathrm{eV}$ [70]. The recent results $[25,70]$ suggest the value closer to $1.0 \mathrm{eV}$. Therefore hydrogen is unlikely to diffuse fast into $\mathrm{HfO}_{2}$ films, as has also been noted in Ref. [66] in the case of deuterium. We note in passing that the wide spread of experimental data for activation energies of hydrogen diffusion in $\mathrm{ZrO}_{2}$ could be understood by assuming that in some samples hydrogen can donate electron to structural defects and move as a proton 
and in some samples it diffuses as an atom. This can happen locally and depends on the diffusion path, as observed in Ref. [25].

Thus hole trapping and $\mathrm{H}^{+}$formation is a likely scenario at Fermi level positions below the bottom of the Si conduction band and $\mathrm{H}^{-}$can be formed at higher Fermi levels (see Fig. 2). This is consistent with the observations of positive charge accumulation in Ref. [67]. Moreover, the striking similarity of the positive and negative charging exponential enhancement in the $\mathrm{SiO}_{2} / \mathrm{HfO}_{2}$ stacks on silicon observed when decreasing the $\mathrm{SiO}_{2}$ interlayer thickness (see Fig. 9 in [14]), points towards the common source of charges of opposite polarity. Their generation by electron or hole tunneling to hydrogen from electrodes is consistent with our results taking into account the well-documented valence band offset between $\mathrm{Si}$ and $\mathrm{HfO}_{2}$ of $2.5 \mathrm{eV}[17,63]$ (see Fig. 2). A positive or negative charged state will be created depending on the orientation of electric field externally applied across the a- $\mathrm{HfO}_{2}$ film provided the diffusion of atomic hydrogen is slow enough to allow for electron tunneling to the electrode or trapping.

\section{ACKNOWLEDGMENTS}

M.K. and A.L.S. are grateful to the World Premier International Research Center Initiative (WPI) sponsored by the Ministry of Education, Culture, Sports, Science and Technology (MEXT), Japan for financial support. A.L.S. acknowledges funding provided by EPSRC under Grant No. EP/K01739X/1. Computer facilities on Archer service have been provided via the UKs HPC Materials Chemistry Consortium (EPSRC Grant No. EP/L000202). The authors thank the Supercomputer Center, the Institute for Solid State Physics, the University of Tokyo for the facilities and the use of the SGI Altix ICE 8400 EX. The authors wish to thank A.-M. El-Sayed, O. Dicks, and J. Strand for helpful discussions.
[1] M. Al-Kuhaili, Opt. Mater. 27, 383 (2004).

[2] J. Khoshman, A. Khan, and M. Kordesch, Surf. Coat. Technol. 202, 2500 (2008).

[3] C. Zhao, C. Z. Zhao, M. Werner, S. Taylor, and P. R. Chalker, ISRN Nanotechnol. 2012, 689023 (2012).

[4] E. Amat, T. Kauerauf, R. Rodríguez, M. Nafría, X. Aymerich, R. Degraeve, and G. Groeseneken, Microelectron. Eng. 103, 144 (2013).

[5] S. Clima, Y. Chen, A. Fantini, L. Goux, R. Degraeve, B. Govoreanu, G. Pourtois, and M. Jurczak, IEEE Electron Device Lett. 36, 769 (2015).

[6] M. Al-Kuhaili, S. Durrani, I. Bakhtiari, M. Dastageer, and M. Mekki, Mater. Chem. Phys. 126, 515 (2011).

[7] S. Kim, D. Lee, J. Park, S. Jung, W. Lee, J. Shin, J. Woo, G. Choi, and H. Hwang, Nanotechnology 23, 325702 (2012).

[8] X. Zou, G. Fang, L. Yuan, X. Tong, and X. Zhao, Semicond. Sci. Technol. 25, 055006 (2010).

[9] F. M. Li, B. C. Bayer, S. Hofmann, J. D. Dutson, S. J. Wakeham, M. J. Thwaites, W. I. Milne, and A. J. Flewitt, Appl. Phys. Lett. 98, 252903 (2011).

[10] J.-H. Kim, J.-W. Kim, J.-H. Roh, K.-J. Lee, K.-M. Do, J.-H. Shin, S.-M. Koo, and B.-M. Moon, Mater. Res. Bull. 47, 2923 (2012).

[11] T. Grasser, Bias Temperature Instability for Devices and Circuits (Springer Science \& Business Media, New York, 2013).

[12] A. Kerber and E. A. Cartier, IEEE Trans. Device Mater. Reliab. 9, 147 (2009).

[13] E. Cartier, A. Kerber, T. Ando, M. Frank, K. Choi, S. Krishnan, B. Linder, K. Zhao, F. Monsieur, J. Stathis et al., in International Electron Devices Meeting (IEDM) (IEEE, Piscataway, 2011), pp. 18.4.1-18.4.4 .

[14] T. Ando, Materials 5, 478 (2012).

[15] C. Tang and R. Ramprasad, Phys. Rev. B 81, 161201 (2010).

[16] F. Cerbu, O. Madia, D. Andreev, S. Fadida, M. Eizenberg, L. Breuil, J. Lisoni, J. Kittl, J. Strand, A. Shluger et al., Appl. Phys. Lett. 108, 222901 (2016)

[17] V. Afanas'ev and A. Stesmans, J. Appl. Phys. 102, 081301 (2007).
[18] A. Hartstein and D. Young, Appl. Phys. Lett. 38, 631 (1981).

[19] J. De Nijs, K. Druijf, V. Afanas'ev, E. Van der Drift, and P. Balk, Appl. Phys. Lett. 65, 2428 (1994).

[20] V. V. Afanas'ev and A. Stesmans, Phys. Rev. Lett. 80, 5176 (1998).

[21] V. Afanas'ev and A. Stesmans, Europhys. Lett. 53, 233 (2001).

[22] F. K. McTaggart, Nature (London) 191, 1192 (1961).

[23] J. Godet, P. Broqvist, and A. Pasquarello, Appl. Phys. Lett. 91, 262901 (2007).

[24] Y. A. Mantz and R. S. Gemmen, J. Phys. Chem. C 114, 8014 (2010).

[25] A. G. Marinopoulos, Phys. Rev. B 86, 155144 (2012).

[26] H. Muta, Y. Etoh, Y. Ohishi, K. Kurosaki, and S. Yamanaka, J. Nucl. Sci. Technol. 49, 544 (2012).

[27] M. Youssef and B. Yildiz, Phys. Chem. Chem. Phys. 16, 1354 (2014).

[28] H. Li and J. Robertson, J. Appl. Phys. 115, 203708 (2014).

[29] J. Lyons, A. Janotti, and C. Van de Walle, Microelectron. Eng. 88, 1452 (2011).

[30] E. Cartier and J. Stathis, Microelectron. Eng. 28, 3 (1995).

[31] M. Houssa, G. Pourtois, M. Heyns, and A. Stesmans, J. Phys.: Condens. Matter 17, S2075 (2005).

[32] C. G. Van de Walle and J. Neugebauer, Nature (London) 423, 626 (2003).

[33] H.-M. Benia, P. Myrach, A. Gonchar, T. Risse, N. Nilius, and H.-J. Freund, Phys. Rev. B 81, 241415 (2010).

[34] C. Gionco, M. C. Paganini, E. Giamello, R. Burgess, C. Di Valentin, and G. Pacchioni, Chem. Mater. 25, 2243 (2013).

[35] D. Ricci, C. Di Valentin, G. Pacchioni, P. V. Sushko, A. L. Shluger, and E. Giamello, J. Am. Chem. Soc. 125, 738 (2003).

[36] E. Finazzi, C. Di Valentin, G. Pacchioni, M. Chiesa, E. Giamello, H. Gao, J. Lian, T. Risse, and H.-J. Freund, Chem. Eur. J. 14, 4404 (2008).

[37] A.-M. El-Sayed, M. B. Watkins, T. Grasser, V. V. Afanas'ev, and A. L. Shluger, Phys. Rev. Lett. 114, 115503 (2015).

[38] A.-M. El-Sayed, Y. Wimmer, W. Goes, T. Grasser, V. V. Afanas'ev, and A. L. Shluger, Phys. Rev. B 92, 014107 (2015). 
[39] Y. Wimmer, A.-M. El-Sayed, W. Gös, T. Grasser, and A. L. Shluger, Proc. R. Soc. London, Ser. A 472, 20160009 (2016).

[40] M. Kaviani, J. Strand, V. V. Afanas'ev, and A. L. Shluger, Phys. Rev. B 94, 020103 (2016).

[41] S. Cox, J. Gavartin, J. Lord, S. Cottrell, J. Gil, H. Alberto, J. P. Duarte, R. Vilao, N. A. De Campos, D. Keeble et al., J. Phys.: Condens. Matter 18, 1079 (2006).

[42] K. Xiong, J. Robertson, and S. Clark, J. Appl. Phys. 102, 083710 (2007).

[43] S. Cox, J. Phys.: Condens. Matter 15, R1727 (2003).

[44] A. Marinopoulos, J. Phys.: Condens. Matter 26, 025502 (2013).

[45] G. Broglia, G. Ori, L. Larcher, and M. Montorsi, Modell. Simul. Mater. Sci. Eng. 22, 065006 (2014).

[46] S. Plimpton, J. Comput. Phys. 117, 1 (1995).

[47] M. Guidon, J. Hutter, and J. VandeVondele, J. Chem. Theory Comput. 5, 3010 (2009).

[48] J. VandeVondele, M. Krack, F. Mohamed, M. Parrinello, T. Chassaing, and J. Hutter, Comput. Phys. Commun. 167, 103 (2005).

[49] G. Lippert, J. Hutter, and M. Parrinello, Mol. Phys. 92, 477 (1997).

[50] J. VandeVondele and J. Hutter, J. Chem. Phys. 127, 114105 (2007).

[51] S. Goedecker, M. Teter, and J. Hutter, Phys. Rev. B 54, 1703 (1996).

[52] S. Lhostis, C. Gaumer, C. Bonafos, S. Schamn, N. Cherkashin, F. Pierre, A. Fanton, C. Morin, F. Ferrieu, M. Casse et al., ECS Transactions 13, 101 (2008).

[53] T. Wang and J. G. Ekerdt, Chem. Mater. 21, 3096 (2009).

[54] J. H. Jang, H.-S. Jung, J. H. Kim, S. Y. Lee, C. S. Hwang, and M. Kim, J. Appl. Phys. 109, 023718 (2011).
[55] A.-M. El-Sayed, M. B. Watkins, V. V. Afanas'ev, and A. L. Shluger, Phys. Rev. B 89, 125201 (2014).

[56] S. Lany and A. Zunger, Modell. Simul. Mater. Sci. Eng. 17, 084002 (2009).

[57] S. Lany and A. Zunger, Phys. Rev. B 78, 235104 (2008).

[58] C. Driemeier, R. Wallace, and I. Baumvol, ECS Transactions 11, 91 (2007).

[59] T. S. Bjørheim, E. A. Kotomin, and J. Maier, J. Mater. Chem. A 3, 7639 (2015).

[60] T. S. Bjørheim, M. Arrigoni, S. W. Saeed, E. Kotomin, and J. Maier, Chem. Mater. 28, 1363 (2016).

[61] D. Gryaznov, R. Merkle, E. Kotomin, and J. Maier, J. Mater. Chem. A 4, 13093 (2016).

[62] S. F. J. Cox, S. P. Cottrell, J. S. Lord, R. C. Vilão, H. V. Alberto, J. P. Duarte, J. Gil, N. A. de Campos, E. Davis, M. Charlton et al., Phys. Semi.; Part A 772, 193 (2005).

[63] V. V. Afanas'ev, Adv. Condens. Matter Phys. 2014, 301302 (2014).

[64] S. Srivastava and N. Sathyamurthy, J. Phys. Chem. A 118, 6343 (2014).

[65] D. Muñoz Ramo, A. L. Shluger, J. L. Gavartin, and G. Bersuker, Phys. Rev. Lett. 99, 155504 (2007).

[66] C. Driemeier, L. Miotti, I. J. R. Baumvol, C. Radtke, E. P. Gusev, M. J. Kim, and R. M. Wallace, Appl. Phys. Lett. 88 041918 (2006).

[67] M. Houssa, S. D. Gendt, J. Autran, G. Groeseneken, and M. Heyns, Appl. Phys. Lett. 85, 2101 (2004).

[68] M. Houssa, V. Afanas'ev, A. Stesmans, and M. Heyns, Appl. Phys. Lett. 79, 3134 (2001).

[69] V. Afanas'ev and A. Stesmans, J. Appl. Phys. 95, 2518 (2004).

[70] D. Khatamian, in Defect and Diffusion Forum, Vol. 297 (Trans Tech Publications, Zurich, 2010), pp. 631-640.

[71] D. Duncan, B. Magyari-Köpe, and Y. Nishi, Appl. Phys. Lett. 108, 043501 (2016). 